\title{
COVERING PROPERTIES OF EXTREMAL VERTICAL SLIT MAPPINGS
}

\author{
By Fumio MaItANI
}

Dedicated to Professor Kôtaro Oikawa on his sixtieth birthday

\section{Introduction.}

Bergman kernels are fundamental on Riemann surfaces and many investigations are given. N. Suita, in his paper [13], gave a formula between Bergman kernels and capacities. J. Lewittes pointed out the following in [8]. If the Gaussian curvature of Bergman metric vanishes at a point on a compact Riemann surface, the surface is hyper-elliptic and the point is one of the Weierstrass points. We shall investigate these theorems from another point of view. We gave variational formulas of meromorphic differentials with certain boundary behavior under a quasiconformal deformation (cf. [9]). In this paper, meromorphic differentials whose real parts are like differentials of potentials and harmonic measures are treated. The functions whose differentials have the boundary behaviors can be called slit mappings and have certain extremal property. The variational formulas of the potentiallike differentials under a trivial quasiconformal deformation shall give above mentioned Suita's formula. Further, using them and Rodin's Riemann-Roch theorem [10], we obtain Lewittes' theorem on an open Riemann surface of infinite genus. Finally we refer to Kusunoki's meromorphic functions whose real parts are like harmonic measures in a neighbourhood of the ideal boundary. In the case of planar surfaces it gives extremal vertical slit mappings. The image region called extremal slit region has been studied by many people since Köbe introduced (cf. [11]). Recently the covering appearance by the extremal slit mapping on a Riemann surface of finite genus are precisely grasped by M. Shiba [12]. However, in the case of infinite genus it remains misty. Is the extremal slit mapping with $n$-poles almost $n$-valent on a Riemann surface of infinite genus? We remark that it is affirmative if Kerékjártó-Stoïlow's boundary components are countable.

The author expresses his thanks to the referee for valuable suggestions.

Received January 27, 1988 


\section{$\S 1$. Preliminary results of variational formulas under quasiconformal deformation.}

We make use of the variational formula of certain meromorphic differentials. It is obtained in [9], where the concerned Riemann surface is deformed holomorphically with complex parameter. Let $R$ be an arbitrary Riemann surface, $\left\{R^{t}\right\}\left(R^{0}=R\right)$ be Riemann surfaces with complex parameter $t=u+i v$ which varies about 0 and $h_{t}$ be a quasiconformal mapping from $R$ to $R^{t}$ whose Beltrami coefficient $\mu(z, t)=\frac{\left(h_{t}\right)_{\bar{z}}}{\left(h_{t}\right)_{z}}$ satisfies the following condition $*$ :

i . $\mu(z, t)$ is measurable and $\mu(z, 0)=0$,

* ii. $\quad \operatorname{esssup}|\mu(z, t+\varepsilon)-\mu(z, t)| \leqq M_{t}|\varepsilon|$,

iii. $\mu(z, t)$ is holomorphic with respect to $t$.

Let $\Lambda$ be the real Hilbert space of square integrable complex differentials whose inner product is given by

$$
\langle\sigma, \omega\rangle=\text { Real part of } \iint \sigma \wedge * \bar{\omega}=\operatorname{Re}(\sigma, \omega),
$$

where $* \omega$ denotes the harmonic conjugate differential of $\omega$ and $\bar{\omega}$ denotes the complex conjugate of $\omega$. The following subspaces of $\Lambda$ will be used

$$
\begin{aligned}
& \Lambda_{h}=\{\lambda \in A: \lambda \text { is a complex harmonic differential }\}, \\
& \Lambda_{e o}=\left\{\lambda \in A: \lambda \text { is a closed differential which is orthogonal to } \Lambda_{h}\right\}, \\
& \Gamma_{h}=\left\{\lambda \in \Lambda_{h}: \lambda \text { is a real differential }\right\}
\end{aligned}
$$

We are concerned with a subspace represented by $\Lambda_{x}=\Gamma_{x}+i^{*} \Gamma_{x}{ }^{\perp}$, where $\Gamma_{x}$ is a subspace of $\Gamma_{h}$ and $* \Gamma_{x}{ }^{\perp}=\left\{\omega \in \Gamma_{h}:\langle\sigma, * \omega\rangle=0\right.$ for any $\left.\sigma \in \Gamma_{x}\right\}$. For a $p^{t} \in R^{t}$, take a parametric disk $V^{t}$ about $p^{t}$ with local variable $z$. There exists a meromorphic differential $\phi_{n, x}^{t}$ with given singularity $\frac{d z}{z^{n+1}}$ at $p^{t}$ which coincides with an element in $\Lambda_{x}+\Lambda_{e o}$ on $R^{t}-V^{t}$ (cf. [9]). If $\phi_{n, x}^{t}$ satisfies the same condition, $\phi_{n, x}^{t}-\phi_{n, x}^{t}$ is holomorphic and belongs to $\Lambda_{x}+\Lambda_{e o}$. Therefore $\phi_{n, x}^{t}-\phi_{n, x}^{t}$ $=i^{*}\left(\phi_{n, x}^{t}-\phi_{n, x}^{t}\right) \in\left(\Lambda_{x}+\Lambda_{e o}\right) \cap\left(i^{*} \Lambda_{x}+* \Lambda_{e o}\right)=\{0\}$. Hence such a $\phi_{n, x}^{t}$ is uniquely determined. When $h_{t}$ is conformal from $V^{0}$ to $V^{t}$ and $\phi_{n, x}^{t}$ is regarded as having the singularity with respect to the same local variable, $\phi_{n, x}^{t} h_{t}-\phi_{n, x}^{t}$ belongs to $\Lambda_{x}+\Lambda_{e o}$, where $\Lambda_{x}\left(R^{t}\right) \circ h_{t} \subseteq \Lambda_{x}(R)+\Lambda_{e o}$. For meromorphic differentials $\left\{\phi^{t}\right\}$ such that $\phi^{t} \circ h_{t}-\phi^{0} \in \Lambda_{x}+\Lambda_{e o}$, we have shown that

$$
\frac{\phi^{t+u} \circ h_{t+u^{\circ}} h_{t}^{-1}-\phi^{t}}{u}\left(\operatorname{resp} . \frac{\phi^{t+\imath v_{\circ}} h_{t+\imath v^{\circ}} h_{t}^{-1}-\phi^{t}}{v}\right)
$$

converges to an element $\left(\phi^{t}\right)_{u}$ (resp. $\left.\left(\phi^{t}\right)_{v}\right)$ in $\Lambda_{x}+\Lambda_{e o}$ in the norm sense as real $u$ (resp. $v$ ) tends to zero. Set $\left(\phi^{t}\right)_{t}=\left(\left(\phi^{t}\right)_{u}+i\left(\phi^{t}\right)_{v}\right) / 2$ and $\left(\phi^{t}\right)_{t}=\left(\left(\phi^{t}\right)_{u}-i\left(\phi^{t}\right)_{v}\right) / 2$. The $\left(\phi^{t}\right)_{\bar{t}}$ becomes a holomorphic differential. We have the following variational 
formulas.

THEOREM A. ([9] Theorem 2, 3, 4, 5) Let meromorphic differentıals $\phi^{t}, \phi^{t}$ satisfy that the poles of $\phi^{0}, \phi^{0}$ do not meet the support of $\mu$ and $\phi^{t} \cdot h_{t}-\phi^{t}$, $\phi^{t} \cdot h_{t}-\psi^{t} \in \Lambda_{x}+\Lambda_{e o}$. Then

$$
\begin{aligned}
& \frac{\partial}{\partial t}\left\langle\phi^{t} \circ h_{t}-\phi^{0}, \overline{\phi^{0}}\right\rangle=\frac{1}{2}\left(\left(\phi^{t}\right)_{t}, \overline{\phi^{t}}\right) \\
& \frac{\partial^{2}}{\partial \bar{t} \partial t}\left\langle\phi^{t} \circ h_{t}-\phi^{0}, \overline{\phi^{0}}\right\rangle=\frac{1}{2}\left\{\left(\left(\phi^{t}\right)_{\bar{t}},\left(\overline{\left.\psi^{t}\right)_{t}}\right)+\left(\left(\phi^{t}\right)_{\bar{t}},\left(\overline{\left.\phi^{t}\right)_{t}}\right)\right\} .\right.\right.
\end{aligned}
$$

Further, when $\phi^{t}, \phi^{t}$ are holomorphic and $\Lambda_{x}=i \Gamma_{h}$,

$$
\begin{aligned}
& \frac{\partial}{\partial t}\left\langle\phi^{t} \circ h_{t}-\phi^{0}, \overline{\phi^{0}}\right\rangle=-\frac{1}{2}\left(\phi^{t},\left(\phi^{t}\right)_{\bar{t}}\right), \\
& \frac{\partial^{2}}{\partial \bar{t} \partial t}\left\langle\phi^{t} \circ h_{t}-\phi^{0}, \overline{\phi^{0}}\right\rangle=-\left\langle\left(\phi^{t}\right)_{\bar{t}},\left(\phi^{t}\right)_{\bar{t}}\right\rangle .
\end{aligned}
$$

In particular,

$$
\begin{aligned}
& \frac{\partial}{\partial t}\left\langle\phi^{t}, \phi^{t}\right\rangle=-\frac{1}{2}\left\{\left(\phi^{t},\left(\phi^{t}\right)_{\bar{t}}\right)+\left(\phi^{t},\left(\phi^{t}\right)_{\bar{t}}\right)\right\} \\
& \frac{\partial^{2}}{\partial \bar{t} \partial t}\left\langle\phi^{t}, \phi^{t}\right\rangle=2\left\langle\left(\phi^{t}\right)_{\bar{t}},\left(\phi^{t}\right)_{\bar{t}}\right\rangle .
\end{aligned}
$$

\section{§. Variation of the reference point of a reproducing differential.}

We consider a specific kind of quasiconformal self mappings on $R$ which represent small displacement of a parametric disk. Take a point $p$ on $R$ and a parametric disk $V=\{z:|z|<2\}$ about $p$. The local parameter $z$ also denotes, for covenience, the corresponding point on $R$. Consider a function $h(z, t)$ on $V$ :

where $|t|<1 / 2$.

$$
h(z, t)= \begin{cases}z+t & |z| \leqq 1 / 2 \\ 2 t(1-|z|)+z & 1 / 2<|z|<1 \\ z & 1 \leqq|z|\end{cases}
$$

Put

$$
h_{t}= \begin{cases}h(z, t) & \text { on } V \\ \text { identity } & \text { on } R-V .\end{cases}
$$

Then $h_{t}$ is a quasiconformal self mapping on $R$ and the Beltrami coefficient is 


$$
\mu(z, t)=\frac{\left(h_{t}\right)_{\bar{z}}}{\left(h_{t}\right)_{z}}=\left\{\begin{array}{cl}
0 & |z| \leqq 1 / 2 \\
\frac{-t z}{|z|-t \bar{z}} & 1 / 2<|z|<1 \\
0 & 1 \leqq|z| \text { and } R-V .
\end{array}\right.
$$

This satisfies the condition $*$.

Now let $\phi_{0}^{t}=d g_{t}+i^{*} d g_{t}$, where $g_{t}$ is a Green function on a hyperbolic Riemann surface $R$ with pole at $t$. For $n \geqq 1$ there exists a meromorphic differential $\phi_{n}^{t}$ (resp. $\psi_{n}^{t}$ ) on an arbitrary Riemann surface $R$ such that

1. $\phi_{n}^{t}-\frac{d z}{(z-t)^{n+1}}\left(\operatorname{resp} . \phi_{n}^{t}-\frac{d z}{(z-t)^{n+1}}\right)$ is a holomorphic differential on $V$,

2. $\phi_{n}^{t}$ coincides with an element in $i \Gamma_{h}+\Lambda_{e o}$ on $R-V$

(resp. $\phi_{n}^{t}$ coincides with an element in $\Gamma_{h}+\Lambda_{e o}$ on $R-V$ ).

It follows that $\phi_{n}^{t} \circ h_{t}-\phi_{n}^{0} \in i \Gamma_{h}+\Lambda_{e o}, \quad \phi_{n}^{t} \circ h_{t}-\phi_{n}^{n} \in \Gamma_{h}+\Lambda_{e o}$. We have the following.

LEMMA 1.

$$
\left(\phi_{n}^{t}\right)_{u}=(n+1) \phi_{n+1}^{t} \text { on } R-V \text { and }\left(\phi_{n}^{t}\right)_{v}=i(n+1) \phi_{n+1}^{t} \text { on } R-V .
$$

Proof. Put

$$
\begin{aligned}
& \lambda_{u}=\frac{\phi_{n}^{t+u}-\phi_{n}^{t}}{u}-\frac{1}{u}\left(\frac{1}{(z-t-u)^{n+1}}-\frac{1}{(z-t)^{n+1}}\right) d z \text { on } V, \\
& \tilde{\lambda}_{v}=\frac{\phi_{n}^{t+\imath v}-\phi_{n}^{t}}{v}-\frac{1}{v}\left(\frac{1}{(z-t-\imath v)^{n+1}}-\frac{1}{(z-t)^{n+1}}\right) d z \text { on } V .
\end{aligned}
$$

Then $\lambda_{u}$ and $\tilde{\lambda}_{v}$ are holomorphic differential on $V$. Remark that $h_{t+u} \cdot h_{t}{ }^{-1}$ and $h_{t+\imath v} \cdot h_{t}{ }^{-1}$ are the identity on $|z|>1$ and on $R-V$. The $\lambda_{u}$ and $\tilde{\lambda}_{v}$ converge to holomorphic differentials $\left(\phi_{n}^{t}\right)_{u}-\frac{n+1}{(z-t)^{n+2}} d z$ and $\left(\phi_{n}^{t}\right)_{v}-\frac{i(n+1)}{(z-t)^{n+2}} d z$ on $|z|>1$ respectively as $u, v \rightarrow 0$, hence they converge on $V$ in the norm sense. Further $\left(\phi_{n}^{t+u}-\phi_{n}^{t}\right) / u$ and $\left(\phi_{n}^{t+v v}-\phi_{n}^{t}\right) / v$ converge to $\left(\phi_{n}^{t}\right)_{u}$ and $\left(\phi_{n}^{t}\right)_{v}$ on $R-V$ respectively and they coincide with elements in $i \Gamma_{h}+\Lambda_{e o}$ on $R-V$. Therefore $\left(\phi_{n}^{t+u}-\phi_{n}^{t} / u\right.$ (resp. $\left.\left(\phi_{n}^{t+\imath v}-\phi_{n}^{t}\right) / v\right)$ converges to $(n+1) \phi_{n+1}^{t}$ (resp. $\left.i(n+1) \phi_{n+1}^{t}\right)$ on $R-\{z=t\}$ as real $u$ (resp. $v$ ) tends to zero. Thus we obtain the results.

As for $\phi_{n}^{t}$, similarly we have

\section{LEMMA 2.}

$$
\left(\phi_{n}^{t}\right)_{u}=(n+1) \phi_{n+1}^{t} \text { on } R-V \text { and }\left(\psi_{n}^{t}\right)_{v}=i(n+1) \phi_{n+1}^{t} \text { on } R-V .
$$

By these Lemmas we have directly 
$\left(\phi_{n}^{t}\right)_{\bar{t}}=\frac{n+1}{2}\left(\phi_{n+1}^{t}-\phi_{n+1}^{t}\right)$ on $R-V$ and $\left(\phi_{n}^{t}\right)_{\bar{t}}=\frac{n+1}{2}\left(\phi_{n+1}^{t}-\phi_{n+1}^{t}\right)$ on $R-V$.

Since $\left(\phi_{n}^{t}\right)_{\bar{t}}$ and $\left(\phi_{n}^{t}\right)_{\bar{t}}$ are holomorphic differentials on $R$, we have $\left(\phi_{n}^{t}\right)_{\bar{t}}=$ $\frac{n+1}{2}\left(\phi_{n+1}^{t}-\phi_{n+1}^{t}\right)=-\left(\phi_{n}^{t}\right)_{\bar{t}}$ on $R$. Now put $K_{n}^{t}=\frac{n !}{4 \pi}\left(\phi_{n}^{t}-\phi_{n}^{t}\right)$ for $n \geqq 1$. The $K_{n}^{t}$ has a reproducing property.

LEMMA 3.

$\left(\omega, K_{n}^{t}\right)=\frac{d^{n}}{d z^{n}} w(t)$ for every holomorphic differential $\omega$ in $\Lambda$,

where $\omega=d w$ on $V$.

Proof. Let the real part of $\phi_{n}^{t}$ (resp. the imaginary part of $\phi_{n}^{t}$ ) coincides with a real differential $\sigma$ (resp. $\tau) \in \Lambda_{e o}$ on $R-V$. Then $\phi_{n}^{t}=\sigma+i^{*} \sigma$ on $R-V$ and

$$
\left(\omega, \phi_{n}^{t}\right)=\left(\omega, \sigma+i^{*} \sigma\right)_{R}-\left(\omega, \sigma+i^{*} \sigma\right)_{V}+\left(\omega, \phi_{n}^{t}\right)_{V},
$$

where principal integral is used. The first term vanishes, and

$$
\begin{gathered}
\left(\boldsymbol{\omega}, \boldsymbol{\sigma}+i^{*} \boldsymbol{\sigma}\right)_{V}=(* \boldsymbol{\omega}, * \boldsymbol{\sigma})_{V}-i(\boldsymbol{\omega}, * \boldsymbol{\sigma})_{V}=-2 i(\boldsymbol{\omega}, * \boldsymbol{\sigma})_{V} \\
=2 i \iint_{V} d w \wedge \boldsymbol{\sigma}=2 i \int_{\partial V} w \operatorname{Re} \boldsymbol{\phi}_{n}^{t}, \\
\left(\boldsymbol{\omega}, \boldsymbol{\phi}_{n}^{t}\right)_{V}=\lim _{\varepsilon \rightarrow 0} \iint_{V-V_{\varepsilon}(t)} d w \wedge * \bar{\phi}_{n}^{t} \\
=\lim _{\varepsilon \rightarrow 0} i \int_{\partial\left(V-V_{\varepsilon}(t)\right)} w \bar{\phi}_{n}^{t}=i \int_{\partial V} w \bar{\phi}_{n}^{t},
\end{gathered}
$$

where $V_{\varepsilon}(t)=\{z ;|z-t|<\varepsilon\}$.

Therefore

$$
\begin{aligned}
\left(\boldsymbol{\omega}, \phi_{n}^{t}\right)_{V}-\left(\boldsymbol{\omega}, \sigma+i^{*} \sigma\right)_{V} & =i \int_{\partial V} w \bar{\phi}_{n}^{t}-2 i \int_{\partial V} w \operatorname{Re} \phi_{n}^{t} \\
& =-i \int_{\partial V} w \phi_{n}^{t}=\frac{2 \pi}{n !} \frac{d^{n} w}{d z^{n}}(t) .
\end{aligned}
$$

On the other hand,

$$
\begin{aligned}
\left(\omega, \phi_{n}^{t}\right) & =\left(\omega,-*^{*}+i \tau\right)_{R}-\left(\omega,-*^{*}+i \tau\right)_{v}+\left(\omega, \phi_{n}^{t}\right)_{0} \\
& =2\left(\omega,{ }^{*}\right)_{V}+\left(\omega, \phi_{n}^{t}\right)_{V}=-2 \int_{\partial V} w \operatorname{Im} \phi_{n}^{t}+\int_{\partial V} w^{*} \bar{\psi}_{n}^{t} \\
& =i \int_{\partial V} w \psi_{n}^{t}=-\frac{2 \pi}{n !} \frac{d^{n} w}{d z^{n}}(t)
\end{aligned}
$$


Thus $\left(\omega, K_{n}^{t}\right)=\frac{d^{n} w}{d z^{n}}(t)$.

We write $\left(K_{n}^{t}\right)_{\bar{t}}=\frac{n !}{4 \pi}\left(\left(\phi_{n}^{t}\right)_{\bar{t}}-\left(\phi_{n}^{t}\right)_{\bar{t}}\right)$. It follows that

$$
\left(K_{n}^{t}\right)_{\bar{t}}=\frac{(n+1) !}{4 \pi}\left(\phi_{n+1}^{t}-\phi_{n+1}^{t}\right)=K_{n+1}^{t} .
$$

For a Robin constant

$$
\gamma(t)=\frac{1}{2 \pi} \int_{|z|=1} g_{t}|d z|
$$

we have

$$
\gamma(t)-\gamma(0)=\left\langle\phi_{0}^{t} \circ h_{t}-\phi_{0}^{t}, \bar{\phi}_{0}^{t}\right\rangle / 2 \pi .
$$

It follows by Theorem $\mathrm{A}$ that

$$
\frac{\partial^{2}}{\partial \bar{t} \partial t} \gamma(t)=\frac{-1}{2 \pi}\left(\left(\phi_{0}^{t}\right)_{\bar{t}},\left(\phi_{0}^{t}\right)_{\bar{t}}\right) .
$$

Since $K_{1}^{t}=\left(\phi_{1}^{t}-\phi_{1}^{t}\right) / 4 \pi=\left(\phi_{0}^{t}\right)_{t} / 2 \pi$ is the Bergman kernel from Lemma 3, we have the following Suita's Theorem

THEOREM. [13]

$$
\frac{\partial^{2}}{\partial \bar{t} \partial t} \gamma(t)=-2 \pi\left(K_{1}^{t}, K_{1}^{t}\right)
$$

For $K_{n}(t)=\left(K_{n}^{t}, K_{n}^{t}\right)$ similar forms are established. The formulas which are shown as Formula (9) in [9] for $n=1$ are settled for every natural number $n$. As the proof was not given in [9], we note it here.

THEOREM 1. [9]

$$
\frac{\partial}{\partial t} K_{n}(t)=\left(K_{n}^{t},\left(K_{n}^{t}\right)_{\bar{t}}\right)
$$

Proof. By Theorem A

$$
\begin{aligned}
\left(\frac{4 \pi}{n !}\right)^{2}\left(K_{n}^{t},\left(K_{n}^{t}\right)_{\bar{t}}\right)= & \left(\phi_{n}^{t}-\phi_{n}^{t},\left(\phi_{n}^{t}\right)_{\bar{t}}\right)-\left(\phi_{n}^{t}-\phi_{n}^{t},\left(\phi_{n}^{t}\right)_{\bar{t}}\right) \\
= & -2 \frac{\partial}{\partial t}\left\{\left\langle\phi_{n}^{t} \circ h_{t}-\phi_{n}^{n}, \bar{\phi}_{n}^{0}\right\rangle+i\left\langle i \psi_{n}^{t} \circ h_{t}-i \psi_{n}^{0}, \overline{\phi_{n}^{0}}\right\rangle\right. \\
& \left.-i\left\langle\phi_{n}^{t} \circ h_{t}-\phi_{n}^{0}, \overline{i \psi_{n}^{0}}\right\rangle+\left\langle i \psi_{n}^{t} \circ h_{t}-i \psi_{n}^{0}, \overline{i \psi_{n}^{0}}\right\rangle\right\} .
\end{aligned}
$$

Write $\phi_{n}^{t}=d \Phi_{n}^{t}$ on $V$ and $\phi_{n}^{0}=i \sigma+\sigma_{0}, \sigma \in \Gamma_{h}, \sigma_{0} \in \Lambda_{e o}$ on $R-V$. Since $\phi_{n}^{t} \circ h_{t}-\phi_{n}^{0}$ $\in i \Gamma_{h}+\Lambda_{e o}$ and $\phi_{n}^{0}=i^{*} \phi_{n}^{0}=-* \sigma+i^{*} \sigma_{0}$ on $R-V$, we have 


$$
\begin{aligned}
\left\langle\phi_{n}^{t} \circ h_{t}-\phi_{n}^{0}, \bar{\phi}_{n}^{0}\right\rangle & =\left\langle\phi_{n}^{t} \circ h_{t}-\phi_{n}^{0},-{ }^{*} \sigma-i^{*} \bar{\sigma}_{0}\right\rangle_{R-V}+\left\langle\phi_{n}^{t} \circ h_{t}-\phi_{n}^{0}, \bar{\phi}_{n}^{0}\right\rangle_{V} \\
& =-\left\langle\phi_{n}^{t} \circ h_{t}-\phi_{n}^{0},-* \sigma-i^{*} \bar{\sigma}_{0}\right\rangle_{V}+\left\langle\phi_{n}^{t} \circ h_{t}-\phi_{n}^{0}, \bar{\phi}_{n}^{0}\right\rangle_{V} \\
& =-\left\langle\phi_{n}^{t} \circ h_{t}-\phi_{n}^{0},-* \sigma-i^{*} \bar{\sigma}_{0}\right\rangle_{V}+\left\langle\phi_{n}^{t} \circ h_{t}-\phi_{n}^{0}, \bar{\phi}_{n}^{0}\right\rangle_{V-V^{\prime}} \\
& =-\operatorname{Re} \int_{\partial V}\left(\Phi_{n}^{t} \circ h_{t}-\Phi_{n}^{0}\right)\left(\sigma-i \sigma_{0}\right)+\operatorname{Re} \int_{\partial\left(V-V^{\prime}\right)}\left(\Phi_{n}^{t} \circ h_{t}-\Phi_{n}^{0}\right)^{*} \phi_{n}^{0},
\end{aligned}
$$

where $V^{\prime}=\{z:|z|<1 / 3\}$. Clearly $\sigma-i \sigma_{0}=* \phi_{n}^{0}$ on $\partial V$, hence

$$
\left\langle\phi_{n}^{t} \circ h_{t}-\phi_{n}^{0}, \overline{\phi_{n}^{0}}\right\rangle=\operatorname{Re} i \int_{\partial V^{\prime}}\left(\Phi_{n}^{t} \circ h_{t}-\Phi_{n}^{0}\right) \frac{d z}{z^{n+1}}=-\frac{2 \pi}{n !} \operatorname{Re} \frac{d^{n}}{d z^{n}}\left(\Phi_{n}^{t} \circ h_{t}-\Phi_{n}^{0}\right) .
$$

Similarly we have

$$
\begin{aligned}
& \left\langle i \psi_{n}^{t} \circ h_{t}-i \psi_{n}^{0}, \overline{\phi_{n}^{0}}\right\rangle=\frac{-2 \pi}{n !} \operatorname{Re} i \frac{d^{n}}{d z^{n}}\left(\Psi_{n}^{t} \circ h_{t}-\Psi_{n}^{0}\right), \quad\left(d \Psi_{n}^{t}=\psi_{n}^{t}\right) \\
& \left\langle\phi_{n}^{t} \circ h_{t}-\phi_{n}^{0}, \overline{i \psi_{n}^{0}}\right\rangle=\frac{-2 \pi}{n !} \operatorname{Re} i \frac{d^{n}}{d z^{n}}\left(\Phi_{n}^{t} \circ h_{t}-\Phi_{n}^{0}\right), \\
& \left\langle i \psi_{n}^{t} \circ h_{t}-i \psi_{n}^{0}, \overline{i \psi_{n}^{v}}\right\rangle=\frac{2 \pi}{n !} \operatorname{Re} \frac{d^{n}}{d z^{n}}\left(\Psi_{n}^{t} \circ h_{t}-\Psi_{n}^{0}\right)
\end{aligned}
$$

Therefore

$$
\begin{aligned}
\left(K_{n}^{t},\left(K_{n}^{t}\right)_{\bar{t}}\right)= & \frac{n !}{4 \pi} \frac{\partial}{\partial t}\left\{\operatorname{Re} \frac{d^{n}}{d z^{n}}\left(\Phi_{n}^{t} \circ h_{t}-\Phi_{n}^{0}\right)-\operatorname{Re} \frac{d^{n}}{d z^{n}}\left(\Psi_{n}^{t} \circ h_{t}-\Psi_{n}^{0}\right)\right. \\
& \left.+i \operatorname{Im} \frac{d^{n}}{d z^{n}}\left(\Phi_{n}^{t} \circ h_{t}-\Phi_{n}^{0}\right)-\imath \operatorname{Im} \frac{d^{n}}{d z^{n}}\left(\Psi_{n}^{t} \circ h_{t}-\Psi_{n}^{0}\right)\right\} \\
= & \frac{n !}{4 \pi} \frac{\partial}{\partial t} \frac{d^{n}}{d z^{n}}\left(\Phi_{n}^{t} \circ h_{t}-\Psi_{n}^{t} \circ h_{t}\right)=\frac{n !}{4 \pi} \frac{\partial}{\partial t}\left(\phi_{n}^{t}-\psi_{n}^{t}, K_{n}^{t}\right) .
\end{aligned}
$$

Further we have

THEOREM 2. [9]

$$
\begin{aligned}
& \frac{\partial^{2}}{\partial \bar{t} \partial t} K_{n}(t)=\left(\left(K_{n}^{t}\right)_{\bar{t}},\left(K_{n}^{t}\right)_{\bar{t}}\right)=K_{n+1}(t), \\
& \frac{\partial^{2}}{\partial \bar{t} \partial t} \log K_{n}(t)=\frac{1}{K_{n}(t)^{2}}\left\{K_{n+1}(t) K_{n}(t)-\left|\left(K_{n+1}^{t}, K_{n}^{t}\right)\right|^{2}\right\} \geqq 0 .
\end{aligned}
$$

Proof. Remark that

$$
\begin{aligned}
\frac{\partial^{2}}{\partial \bar{t} \partial t} K_{n}(t)=-2\left(\frac{n !}{4 \pi}\right)^{2} \frac{\partial^{2}}{\partial \bar{t} \partial t} & \left\{\left\langle\phi_{n}^{t} \circ h_{t}-\phi_{n}^{0}, \overline{\phi_{n}^{0}}\right\rangle+i\left\langle\imath \psi_{n}^{t} \circ h_{t}-\imath \psi_{n}^{0}, \overline{\phi_{n}^{0}}\right\rangle\right. \\
& \left.-i\left\langle\phi_{n}^{t} \circ h_{t}-\phi_{n}^{0}, \overline{i \psi_{n}^{0}}\right\rangle+\left\langle i \psi_{n}^{t} \circ h_{t}-i \psi_{n}^{t}, \overline{i \psi_{n}^{0}}\right\rangle\right\}
\end{aligned}
$$

By Theorem A, 


$$
\begin{aligned}
\frac{\partial^{2}}{\partial \bar{t} \partial t} K_{n}(t)=2\left(\frac{n !}{4 \pi}\right)^{2}\left\{\left\langle\left(\phi_{n}^{t}\right)_{\bar{t}},\left(\phi_{n}^{t}\right)_{\bar{t}}\right\rangle+i\left\langle\left(i \psi_{n}^{t}\right)_{\bar{t}},\left(\phi_{n}^{t}\right)_{\bar{t}}\right\rangle\right. \\
\left.-i\left\langle\left(\phi_{n}^{t}\right)_{\bar{t}},\left(i \phi_{n}^{t}\right)_{\bar{t}}\right\rangle+\left\langle\left(i \psi_{n}^{t}\right)_{\bar{t}},\left(i \psi_{n}^{t}\right)_{\bar{t}}\right\rangle\right\} \\
=2\left(\frac{n !}{4 \pi}\right)^{2}\left\{\left\langle\left(\phi_{n}^{t}\right)_{\bar{t}},\left(\phi_{n}^{t}\right)_{\bar{t}}\right\rangle+\left\langle\left(\phi_{n}^{t}\right)_{\bar{t}},\left(\phi_{n}^{t}\right)_{\bar{t}}\right\rangle\right\} \\
=\left(\frac{n !}{4 \pi}\right)^{2}\left\{\left\langle\left(\phi_{n}^{t}\right)_{\bar{t}}-\left(\phi_{n}^{t}\right)_{\bar{t}},\left(\phi_{n}^{t}\right)_{\bar{t}}-\left(\phi_{n}^{t}\right)_{\bar{t}}\right\rangle\right. \\
\left.+\left\langle\left(\phi_{n}^{t}\right)_{\bar{t}}+\left(\phi_{n}^{t}\right)_{\bar{t}},\left(\phi_{n}^{t}\right)_{\bar{t}}+\left(\phi_{n}^{t}\right)_{\bar{t}}\right\rangle\right\} .
\end{aligned}
$$

Since $\left(\phi_{n}^{t}\right)_{\bar{t}}-\left(\phi_{n}^{t}\right)_{\bar{t}}=4 \pi K_{n+1}^{t} / n !$ and $\left(\phi_{n}^{t}\right)_{\bar{t}}+\left(\phi_{n}^{t}\right)_{\bar{t}}=0$, we obtain

$$
\frac{\partial^{2}}{\partial \bar{t} \partial t} K_{n}(t)=\left(K_{n+1}^{t}, K_{n+1}^{t}\right) \text {. }
$$

The second formula is also obtained.

\section{$\S 3$. The vanishing points of a second variation.}

On a compact Riemann surface J. Lewittes showed that the point at which the Gaussian curvature of the Bergman metric vanishes corresponds to the Weierstrass point on hyper-elliptic Riemann surface. In this section, we shall show that the similar statement holds on open Riemann surface of infinite genus.

For a divisor $\delta$, take the following complex vector spaces of meromorphic functions and differentials.

$M(\delta)=\{f$; i. $f$ is a meromorphic function on $R$ whose divisor is a multiple of $\delta$, ii. the differential $d f$ coincides with an element of $\Lambda_{e o}$ in a connected neighbourhood of the ideal boundary\}.

$D(\delta)=\{\phi ;$ i. $\phi$ is a meromorphic differential whose divisor is a multiple of $\delta$,

ii. it is square integrable in a neighbourhood of the ideal boundary,

iii. it has vanishing periods along cycles which divide a part of the ideal boundary from a compact set containing the support of $\delta\}$.

Remark. These classes are the same in [10] which are given for identity partition. Let $A$ and $B$ be disjoint integral divisors on $R$ and $\delta=B / A$. It is known that

THEOREM B. [10]

$$
\operatorname{dim} M(1 / \delta)=\operatorname{deg} B+1-\min (1, \operatorname{deg} A)-\operatorname{dim} D(1 / A) / D(\delta) .
$$

Let $K_{n}^{t} \neq 0$. The second formula in Theorem 2 shows that $K_{n+1}^{t}$ is a constant multiple of $K_{n}^{t}$ if and only if $\frac{\partial^{2}}{\partial \bar{t} \partial t} \log K_{n}(t)=0$. In this circumstances, for a holomorphic differential $\omega=d w \in \Lambda$, we have $\frac{d^{n+1}}{d z^{n+1}} w(t)=0$ if $\frac{d^{n}}{d z^{n}} w(t)=0$. 
Hence $D\left(t^{n+1}\right)=D\left(t^{n}\right)$. By Theorem B.

$$
\begin{aligned}
& \operatorname{dim} M\left(1 / t^{n}\right)=n+1-\operatorname{dim} D(1) / D\left(t^{n}\right), \\
& \operatorname{dim} M\left(1 / t^{n+1}\right)=n+1+1-\operatorname{dim} D(1) / D\left(t^{n+1}\right)=\operatorname{dim} M\left(1 / t^{n}\right)+1 .
\end{aligned}
$$

This implies that there exists a meromorphic function $f$ in $M\left(1 / t^{n+1}\right)-M\left(1 / t^{n}\right)$. When $K_{n}(t)=0$, every differential in $D\left(t^{n-1}\right)$ has zero of order $n$ at $t$. Hence $D\left(t^{n-1}\right)=D\left(t^{n}\right)$. Thus we have the following.

THEOREM 3.

If $K_{n}(t)=0$, then $M\left(1 / t^{n}\right) \supsetneq M\left(1 / t^{n-1}\right)$.

If $K_{n}(t) \neq 0$ and $\frac{\partial^{2}}{\partial \bar{t} \partial t} \log K_{n}(t)=0$, then $M\left(1 / t^{n+1}\right) \supsetneq M\left(1 / t^{n}\right)$.

Remark. If there exists a meromorphic function $f$ in $M\left(1 / t^{n}\right)-M\left(1 / t^{n-1}\right)$, according to R.D.M. Accola [1] $R$ becomes $n$-sheeted covering surface of the extended complex plane by $f$. The $f$ is constant on the harmonic boundary of Royden's compactification $R^{*}$ of $R$, because Dirichlet potential vanishes on the harmonic boundary (cf. [3]). If $R$ is hyperbolic, then $f$ is constant. This is a contradiction. Hence $R$ is parabolic. By Heins' theorem [5], the $f$ covers just $n$-sheets of the complex plane except for a set of capacity zero. The point $t$ is one of the branch points. Since $\frac{-2}{K_{1}(t)} \frac{\partial^{2}}{\partial t \partial t} \log K_{1}(t)$ is the Gaussian curvature of Bergman metric, we have the following

COROLlARY. [8] Let $R$ be a non-planar Riemann surface. If the Gaussian curvature of the Bergman metric has zero, then $R$ is an (ultra) hyper-elliptic Riemann surface of parabolic type. Conversely, if $R$ is an (ultra) hyper-elliptic Riemann surface of parabolic type, then the branch points coincide with the zeros of the Gaussian curvature of the Bergman metric.

Proof. Since $R$ is a non planar Riemann surface, $K_{1}(t) \neq 0$. If the Gaussian curvature vanishes at $t, \frac{\partial^{2}}{\partial \bar{t} \partial t} \log K_{1}(t)=0$. Then $K_{2}(t)=c K_{1}(t)$ and $D\left(t^{2}\right)=D(t)$. Hence there exists a function in $M\left(1 / t^{2}\right)-M(1 / t)$. The $R$ is an (ultra) hyperelliptic Riemann surface of parabolic type and $t$ is the one of branch points. Conversely, at the branch point $t M\left(1 / t^{2}\right) \supsetneq M(1 / t)$ and $D\left(t^{2}\right)=D(t)$. Hence $K_{2}^{t}=$ $c K_{1}^{t}$ and the Gaussian curvature vanishes at $t$.

Remark. This result can be also obtained from the equality of Lagrange with respect to the Gaussian curvature of Bergman metric.

\section{$\S 4$. A covering surface by an extremal slit mapping.}

Y. Kusunoki [6] formulated the Riemann-Roch theorem which is similar type of above cited theorem $\mathrm{B}$ and is related to extremal slit mappings. Let 
$\Lambda_{1}=\Gamma_{h m}+i \Gamma_{h s e}$, where $\Gamma_{h m}$ denote the subspace of $\Gamma_{h}$ which is called harmonic measure and $\Gamma_{h s e}$ the subspace of harmonic semi exact real differentials (cf. [2]). Consider the following real vector space of functions,
$M_{1}(1 / \delta)=\{f ; f$ is a meromorphic function whose divisor is a multiple of $1 / \delta$ and the differential df coincides with an element of $\Lambda_{1}+\Lambda_{e o}$ in a neighbourhood of the ideal boundary\}.

As we have shown at the end of the last section, a function $f$ in $M\left(1 / t^{n}\right)$ $M\left(1 / t^{n-1}\right)$ is almost $n$-valence. So the following question is natural. Is a function $f$ in $M_{1}\left(1 / p^{n}\right)-M_{1}\left(1 / p^{n-1}\right)$ almost $n$-valence? In the case when $R$ is of finite genus, it is known that $R$ is regarded as almost $n$-sheeted covering surface over the extended complex plane with vertical slits by $f$ (cf. [12]). As for the case when $R$ is of infinite genus, we point out the following.

Proposition. Let $R$ have at most a countable number of Kerékjártó-Stoïlow's boundary components. Then $f$ in $M_{1}\left(1 / p^{n}\right)-M_{1}\left(1 / p^{n-1}\right)$ takes values $n$-times except for a countable number of vertical slits and points.

Proof. Suppose $f$ takes a value $w_{0}$ at least $(n+1)$-times. For $p_{0}, \cdots p_{n} \in$ $f^{-1}\left(w_{0}\right)$ there are neighbourhoods $V_{\imath}$ of $p_{i}$ such that $f$ is conformal on each $V_{\imath}$ to $f\left(V_{\imath}\right)=\left\{w ;\left|w-w_{0}\right|<\varepsilon\right\}$ for sufficiently small $\varepsilon$, where we may assume that $\left\{p_{i}\right\}$ are different each other by proper choice of $w_{0}$. Take a neighbourhood $V$ of $p$ on which $f$ takes each value of $\left\{w ;|w|>N>\left|w_{0}\right|+\varepsilon\right\}$ just $n$-times for sufficiently large $N$. The real part of $f$ (denoted by $\operatorname{Re} f$ ) can be regarded as a quasi-continuous function on Kuramochi's compactification $R^{*}$ of $R$ and it takes a constant value quasi-everywhere on each boundary component of $R^{*}-R$ which corresponds to Kerékjártó-Stoïlow's boundary component (cf. [7]). Let $\operatorname{Re} f$ take the value $c_{k}$ quasi-everywhere on a boundary component $\Delta_{k}$. Since there are only a countable number of boundary components, the set $\Delta(s)$ of boundary points on which $\operatorname{Re} f$ does not take the values $\left\{c_{k}\right\}$ is of Kuramochi capacity zero. Therefore the family of rectifiable curves which converges to points in $\Delta(s)$ from a compact set has an infinite extremal length (cf. [4]). Let $L$ be a family of rectifiable curves $\left\{l_{\alpha}\right\}$ which tends to the ideal boundary from $\cup V_{\imath}$ such that $\cap c l\left\{\operatorname{Re} f(p) ; p \in l_{\alpha} \cap\left(R-R_{m}\right)\right\}$ does not intersect any $c_{k}$, where $\left\{R_{m}\right\}$ is a regular exhaustion of $R$ and $c l$ means closure of the set. The curves $\left\{l_{\alpha}\right\}$ tend to $\Delta(s)$ and the extremal length $\lambda(L)$ must be infinite. Let $l_{u, 2}$ be a level curve of $\operatorname{Re} f$ such that i) $\operatorname{Re} f=u$ on $l_{u, \imath}$, ii) there is no branch point of $f$ on $l_{u, \imath}$, iii) $l_{u, \imath}$ starts from $V_{\imath}$ and $-i d f>0$ along $l_{u, \imath}$. Since $\operatorname{Im} f$ strongly increases along $l_{u, \imath}, l_{u, \imath}$ has no cluster point in $R$ except $p$. There are at most $n$ curves of $\left\{l_{u, \imath}\right\}$ which meet $V$. Hence there exists an $l_{u, \imath}$ which tends to the ideal boundary. If $u \neq c_{k}$ for any $k$, the $l_{u, \imath}$ tends to $\Delta(s)$. We can find such an $l_{u, \imath}$ for each $u$ in $\left(\left|w_{0}\right|-\varepsilon,\left|w_{0}\right|+\varepsilon\right)$ except a countable number of points and we denote it by $l_{u}$. Set $L_{1}=\left\{l_{u}\right\}$. The $L_{1}$ is a subfamily of $L$. We can take $w=f(z)=u+i v$ as a local parameter at a point except branch points. For any 
admissible density $\rho(w)|d w|$ for $L_{1}$, which satisfies that inf $\left\{\int_{l_{u}} \rho|d w| ; l_{u} \in L_{1}\right\}=1$,

$$
\varepsilon \leqq \int_{\left|w_{0}\right|-\varepsilon / 2}^{\left|w_{0}\right|+\varepsilon / 2} d u \leqq \int_{\left|w_{0}\right|-\varepsilon / 2}^{\left|w_{0}\right|+\varepsilon / 2} d u \int_{l_{u}} \rho d v \leqq\left\{\iint_{R-\cup V_{2}-V} \rho^{2} d u d v \iint_{R-\cup V_{i}-V} d u d v\right\}^{1 / 2}
$$

Remark that $\iint_{R-\cup v_{\imath}-V} d u d v<\infty$, because $f$ has a finite Dirichlet integral in a neighbourhood of the ideal boundary. This shows that the extremal length $\lambda\left(L_{1}\right)$ of $L_{1}$ is finite. Hence $\lambda(L)$ is also finite by comparison principle. This is a contradiction. Thus $f$ is at most $n$-valence. Let $A$ be the set of complex numbers which $f$ takes at most $(n-1)$-times. Suppose that there is a component of $A$ whose vertical projection $B$ to the real axis is not a point. Level curves $\operatorname{Re} f=x$ for $x \in B$ are not included in a compact set of $R$, if there is no branch point on them. For almost every $x \in B$, there exists a level curve tends to the ideal boundary. This contradicts, as above argument, that the $\Delta(s)$ is of Kuramochi capacity zero. Thus we have obtained the statement.

\section{REFERENCES}

[1] R. Accola, Some classical theorems on open Riemann surfaces, Bull. Amer. Math. Soc. 73 (1967), 13-26.

[2] L. Ahlfors and L. Sario, Riemann surfaces, Princeton Univ. Press (1960), $382 \mathrm{pp}$.

[3] C. Constantinescu And A. Cornea, Ideale Ränder Riemannscher Flächen, Springer-Verlag (1963), $244 \mathrm{pp.}$

[4] T. Fujne, Extremal length and Kuramoch1 boundary, J. Math. Kyoto Univ. 4 (1964), 149-159.

[5] M. Heins, On certain meromorphic functions of bounded valence, Rev. Math. Pures Appl. 2 (1957), 263-267.

[6] Y. KusunOKI, Theory of Abelian integrals and its applications to conformal mappings, Mem. Coll. Sci. Univ. Kyoto Math. 30 (1959), 235-258. Supplements and corrections, Ibid. 33 (1961), 429-433.

[7] Y. Kusunoki, Characterizations of canonical differentials, J. Math. Kyoto Univ. 5 (1966), 197-207.

[8] J. Lewittes, Differentials and metrics on Riemann surfaces, Trans. Amer. Math. Soc. 139 (1969), 311-318.

[9] F. Maitani, Variations of meromorphic differentials under quasiconformal deformations, J. Math. Kyoto Univ. 24 (1984), 49-66.

[10] B. Rodin AND L. Sario, Principal functions, Van Nostrand, Princeton (1968), $347 \mathrm{pp}$.

[11] L. Sario And K. Oikawa, Capacity Functions, Springer-Verlag (1969), 361 pp.

[12] M. ShibA, The Riemann-Hurwitz relation, parallel slit covering map, and continuation of an open Riemann surface of finite genus, Hiroshima Math. J. 14 (1984), 371-399.

[13] N. Suita, On capacities and kernels of Riemann surfaces, Arch. Rat. Mech. Anal. 46 (1972), 212-217.

Kyoto Institute of Technology 